\title{
Optimization of Immunofluorescent Detection of Bone Marrow Disseminated Tumor Cells
}

\author{
Haley D. Axelrod ${ }^{1,2^{*}}$, Kenneth J. Pienta ${ }^{1}$ and Kenneth C. Valkenburg ${ }^{1}$
}

\begin{abstract}
Background: Cancer metastasis is the primary cause of cancer-related deaths and remains incurable. Current clinical methods for predicting metastatic recurrence are not sensitive enough to detect individual cancer cells in the body; therefore, current efforts are directed toward liquid biopsy-based assays to capture circulating and disseminated tumor cells (CTCs and DTCs) in the blood and bone marrow, respectively. The most promising strategy is fluorescence-based immunostaining using cancer cell-specific markers. However, despite recent efforts to develop robust processing and staining platforms, results from these platforms have been discordant among groups, particularly for DTC detection. While the choice of cancer cell-specific markers is a large factor in this discordance, we have found that markerindependent factors causing false signal are just as critical to consider. Bone marrow is particularly challenging to analyze by immunostaining because endogenous immune cell properties and bone marrow matrix components typically generate false staining. For immunostaining of whole tumor tissue containing ample cancer cells, this background staining can be overcome. Application of fluorescent-based staining for rare cells, however, is easily jeopardized by immune cells and autofluorescence that lead to false signal.
\end{abstract}

Results: We have specifically found two types of background staining in bone marrow samples: autofluorescence of the tissue and non-specific binding of secondary antibodies. We systematically optimized a basic immunofluorescence protocol to eliminate this background using cancer cells spiked into human bone marrow. This enhanced the specificity of automated scanning detection software. Our optimized protocol also outperformed a commercial rare cell detection protocol in detecting candidate DTCs from metastatic patient bone marrow.

Conclusions: Robust optimization to increase the signal-to-noise ratio of immunofluorescent staining of bone marrow is required in order to achieve the necessary sensitivity and specificity for rare cell detection. Background immunofluorescent staining in bone marrow causes uncertainty and inconsistency among investigators, which can be overcome by systematically addressing each contributing source. Our optimized assay eliminates sources of background signal, and is adaptable to automated staining platforms for high throughput analysis.

Keywords: Immunofluorescence, Cancer, CTC, DTC, Bone Marrow, Blood, Detection

\footnotetext{
* Correspondence: haxelro2@jhmi.edu

${ }^{1}$ The James Buchanan Brady Urological Institute, Johns Hopkins University

School of Medicine, 600 North Wolfe St., Marburg Building Room 121,

Baltimore, MD 21287, USA

${ }^{2}$ The Cellular and Molecular Medicine Program, Johns Hopkins School of

Medicine, 600 North Wolfe St., Marburg Building Room 121, Baltimore, MD

21287, USA
}

(c) The Author(s). 2018 Open Access This article is distributed under the terms of the Creative Commons Attribution 4.0 International License (http://creativecommons.org/licenses/by/4.0/), which permits unrestricted use, distribution, and reproduction in any medium, provided you give appropriate credit to the original author(s) and the source, provide a link to the Creative Commons license, and indicate if changes were made. The Creative Commons Public Domain Dedication waiver (http://creativecommons.org/publicdomain/zero/1.0/) applies to the data made available in this article, unless otherwise stated. 


\section{Background}

Approximately 600,000 cancer-related deaths occur in the U.S. every year, and nearly all are due to metastasis [1-3]. Once metastatic, cancer is usually incurable; this has led current efforts to focus on early detection of cancer cells by liquid biopsy. However, the accurate detection and characterization of circulating tumor cells (CTCs) in the blood and disseminated tumor cells (DTCs) in the bone marrow (BM) has proven to be challenging. Despite widespread efforts to design assays with the necessary sensitivity and specificity to capture extremely rare cells (1 CTC per ten million white blood cells) there remains only one FDA-approved imagebased immunofluorescence (IF) platform, limited to CTC detection [4-6]. In addition to IF-based rare cell detection, another widely used strategy is based on real time PCR (RT-PCR). RT-PCR methods do not capture individual cancer cell heterogeneity and rely on RNA expression, while IF assays are not as sensitive. Each strategy bears its own limitations, but IF provides several advantages over RT-PCR detection. IF of blood and BM smears allows for the characterization of individual cells at the protein level, where expression does not always correlate with RNA expression [7]. In addition to being able to analyze the expression of multiple proteins, information on protein localization and cell size, shape, and aggregate behavior can be assessed. Combining these features would provide a more informative landscape of the disease to aid in diagnosis, prognosis, and treatment strategies, and thus IF staining has emerged as the most appealing rare cell detection strategy. However, current IF procedures for staining CTCs and DTCs vary widely and have not yielded consistent results $[4,5]$.

While pathologists successfully use chromogenic immunostaining to detect the presence of cancer in many types of tissue with few limitations, when it comes to detecting rare cells these limitations become unacceptable. Compared to chromogenic staining, IF introduces additional factors that need to be controlled for such as microscope exposure time, brightness and contrast settings, photobleaching, and autofluorescence. Improper control of these factors can lead to false negative or positive signal. These misleading effects can be easily avoided in samples where the target cell population is abundant, but can pose complications when trying to detect rare cells. These problems become further amplified when staining and imaging BM, specifically. BM contains a large number of immune cells which, by nature, bind antibodies and engulf foreign particulates. This is largely facilitated by fragment crystallizable (Fc) receptors on the surface of many immune cells. Therefore, when immune cells are present in the specimen being stained it is crucial that appropriate blocking factors be included that inhibits $F_{c}$ receptor binding, prior to the addition of any antibody. Immune cells also tend to be more autofluorescent than other cell types. This has been observed in large macrophages when exposed to $488 \mathrm{~nm}$ light, due to their high content of flavoproteinassociated granules [8, 9]. Another source of blood and $\mathrm{BM}$ autofluorescence is lipofuscin, a product of oxidized proteins and lipids commonly found in macrophages and red blood cells and which fluoresces in most channels [10-12]. Importantly, the BM contains additional extracellular matrix components such as collagen and other autofluorescent non-collagenous proteins which make IF staining of BM more challenging than blood [13-16]. Current IF protocols for the detection of CTCs and DTCs do not consider all of the aforementioned sources of false signal, and this is likely a major factor in the inconsistency of reports that utilize different procedures. It is crucial to consider these potential sources when trying to identify DTCs. Since bone marrow DTCs have not yet been well characterized, it is difficult to judge true positive signal based on histopathological or protein expression traits. Some rare cell detection strategies rely on filtering out immune cells by size, charge, and/or marker exclusion before being processed onto a slide for staining, but these methods run the risk of occasionally filtering out cancer cells $[4,7,17]$. For techniques that do not involve a physical selection step, thorough optimization has been limited by the constraints of autostaining platforms, which are necessary when processing large volumes to find rare events.

We had initially set out to test various cancer cellspecific markers for the detection of DTCs using several different protocols, but found a remarkable number of epithelial marker-positive cells in cancer-negative control samples, in addition to inconsistencies in overall staining. In order to eliminate these uncertainties and develop a reliable staining protocol, we assessed each step of a basic IF protocol for sources contributing to false signal in BM samples. We used a pan-cytokeratin antibody to detect prostate cancer cells spiked into human BM, as this epithelial marker has been consistently used for rare cancer cell detection across a variety of platforms [18-21]. While we recognize that determining cancer cell-specific markers will be crucial in the accurate detection of rare cells, it is first necessary that the staining procedure results in minimal background and consistency across samples, so that cancer-specific markers can be accurately assessed for their specificity and best signal-to-noise ratio. Initially, we observed two main sources of background in particular: tissue autofluorescence and non-specific binding of secondary antibodies. We then systematically optimized the basic IF protocol to increase signal-to-noise ratio of true staining so as to eliminate background signal and to produce an image in which cancer cells were easily identifiable using 
automated detection software. The ultimate goal of this study was to adapt our optimized protocol for use in automated staining platforms to reliably detect DTCs in clinical samples. While our protocol was optimized using BM samples, we anticipate the staining of blood samples to be of equal or greater quality due to the reduced background staining from the immune cell component.

\section{Methods}

For a comprehensive list of reagent vendors and catalog numbers, see Additional file 1: Table S1.

\section{Cell Culture and Cancer Cell Spiking}

LNCaP prostate cancer cells (ATCC) were maintained in RPMI supplemented with $10 \%$ FBS and $1 \%$ penicillin/ streptomycin. Before spiking into bone marrow, LNCaP cells were seeded with $5 \mathrm{nM}$ R1881 (Sigma, \#R0908) in media with charcoal-stripped FBS for two days. Cells were harvested using non-enzymatic cell dissociation buffer (ThermoFisher, \#13151014), resuspended in media, washed in PBS, and then counted using the Countess II FL Automated Cell Counter (ThermoFisher). LNCaP cells were spiked into bone marrow at 1000 LNCaP cells per 1 . 5 million white blood cells (WBCs). Bone marrow was collected from patients following signed written formal consent approved by the Johns Hopkins Office of Human Subjects Research Institutional Review Board.

\section{Bone Marrow Processing for Adhesion Slides}

Human BM aspirate was collected into CellSave Preservative Tubes (Veridex/Janssen Diagnostics, \#7900005) from the pubic bone of low grade prostate cancer patients at the time of radical prostatectomy (see Additional file 1: Table S2 for specific clinical information). The BM was processed within $48 \mathrm{~h}$ of collection. $5 \mathrm{~mL}$ of aspirate was added to $45 \mathrm{~mL}$ of ACK Lysing Buffer (Quality Biological, \#118-156-101) and incubated on a rotator for $10 \mathrm{~min}$ and then spun down at $1500 \times \mathrm{g}$ for $10 \mathrm{~min}$. Pelleted cells were resuspended in $5 \mathrm{~mL}$ of PBS and counted using a WBC counter $\left(\mathrm{HemoCue}^{\circ}\right)$. A desired volume corresponding to 1.5 million WBCs per square on a Marienfeld Adhesion Slide (Azer Scientific, Inc., \#ES0909101) was spun down again and resuspended in PBS corresponding to $300 \mu \mathrm{L}$ per square. $1000 \mathrm{LNCaP}$ cells were added to spiked samples before spinning down. Resuspended cells were pipetted onto slides and incubated at $37^{\circ} \mathrm{C}$ for $1 \mathrm{~h}$ to promote adhesion to slides. Excess PBS was decanted, and slides were then incubated with $4 \%$ paraformaldehyde (ThermoFisher, \#28908) for either 10 or $30 \mathrm{~min}$. After fixation, slides were washed in PBS before. To dehydrate, slides were incubated in 50\% ethanol for $5 \mathrm{~min}, 70 \%$ ethanol for $5 \mathrm{~min}$, and then $100 \%$ for $5 \mathrm{~min}$. Slides were transferred to storage tubes (Fisher Scientific, \#22-038-399) containing $100 \%$ ethanol and were placed at $-20{ }^{\circ} \mathrm{C}$ for long-term storage. All steps were performed at room temperature unless otherwise indicated.

\section{Bone Marrow Processing for Plus Slides Using the RareCyte System}

Six $\mathrm{mL}$ of BM from metastatic prostate cancer patients (see Additional file 1: Table S3 for specific clinical information) was harvested in RareCyte ${ }^{\circ}$ BM tubes. Each tube was inverted 8 times. Each BM sample was filtered through a $100 \mu \mathrm{M}$ Nylon cell strainer (Falcon, \#352360). WBC counts were performed using the HemoCue ${ }^{\circ}$ WBC counter. $7.5 \mathrm{~mL}$ of $\mathrm{PB}$ or $4 \mathrm{~mL}$ of $\mathrm{BM}$ was pipetted into an AccuCyte ${ }^{\odot}$ Separation Tube, which was then inserted into a centrifuge adapter. Each sample was centrifuged at room temperature for $25 \mathrm{~min}$ at $5,200 \times \mathrm{g}$. Using the CyteSealer, a ring was applied around each Separation Tube. Plasma was saved and stored in $-80{ }^{\circ} \mathrm{C} .4 \mathrm{~mL}$ of AccuCyte ${ }^{\circ}$ Displacement Fluid was placed into the Separation Tube, which was then removed by the insertion of the EpiCollector into the Separation Tube. A locking clip was attached to the Separation Tube. The AccuCyte ${ }^{\circ}$ Shield Tube was removed and replaced with the AccuCyte Isolation Tube. $160 \mu \mathrm{L}$ of AccuCyte ${ }^{\odot}$ Isolation Fluid was pipetted into the Isolation Tube and each sample was centrifuged at room temperature for $20 \mathrm{~min}$ at $1000 \times \mathrm{g}$. The Isolation Tube was removed and $760 \mu \mathrm{L}$ of AccuCyte ${ }^{\circ}$ Transfer Fluid was added to resuspend the pellet in the Isolation Tube. $95 \mu \mathrm{L}$ of the resuspended pellet was spread across 24 Superfrost ${ }^{\circ}$ Plus slides and air-dried. Slides were then stored at $-20^{\circ} \mathrm{C}$.

\section{Thawing Plus Slides for Optimized Protocol Staining}

Frozen plus slides were thawed for $10 \mathrm{~min}$ at room temperature, and then fixed with $4 \%$ paraformaldehyde for $10 \mathrm{~min}$. They were then washed in PBS for $3 \mathrm{~min}$, and then washed in PBST two times for $3 \mathrm{~min}$ each. Staining was then initiated starting with the $0.5 \%$ Triton $\mathrm{X}-100$ permeabilization step in the optimized protocol.

\section{Basic Staining Protocol}

All steps were performed at room temperature unless otherwise indicated. All reagents were applied by gently pipetting $300 \mu \mathrm{L}$ onto each square of the Adhesion slide and placing in a humidity chamber. First, stored slides were rehydrated by incubating in $70 \%$ ethanol for $5 \mathrm{~min}$, $50 \%$ ethanol for $5 \mathrm{~min}$, then PBS for $10 \mathrm{~min}$. Next, cells were permeabilized with $0.5 \%$ Triton X-100 diluted in PBS for $20 \mathrm{~min}$. Slides were then washed by dipping into fresh PBS three times and blocked by adding 10\% goat serum for $20 \mathrm{~min}$ at $37{ }^{\circ} \mathrm{C}$. Primary antibodies were diluted in $10 \%$ goat serum and applied to slides for $30 \mathrm{~min}$ at $37{ }^{\circ} \mathrm{C}$ after decanting block. Slides were washed in PBS three times, incubated in secondary antibodies diluted 1:1000 in PBS for $30 \mathrm{~min}$ at $37^{\circ} \mathrm{C}$ in the dark, and 
washed another three times. Finally, glass coverslips were mounted onto the slides using DAPI-containing mounting media, and were left to cure overnight in the dark.

\section{Optimized Staining Protocol}

All steps were performed at room temperature unless otherwise indicated. All reagents were applied by gently pipetting $300 \mu \mathrm{L}$ onto each square of the Adhesion slide and placing in a humidity chamber. Stored slides were rehydrated by incubating in $70 \%$ ethanol for $5 \mathrm{~min}, 50 \%$ ethanol for $5 \mathrm{~min}$, then PBS for $5 \mathrm{~min}$. Thorough washing was performed by placing slides in fresh PBST two times for 3 min each.

Cells were permeabilized by incubating in $0.5 \%$ Triton X-100 diluted in PBS for $20 \mathrm{~min}$. Slides were washed two times in PBST as indicated above, and one time in PBS. For the first blocking step slides were exposed to TrueBlack $^{\text {Tix }}$ diluted fresh in $70 \%$ ethanol for $1 \mathrm{~min}$, then washed in PBS two times and in PBST one time. Additional blocking steps were performed by incubating slides in Image-iT ${ }^{\mathrm{mm}}$ FX Signal Enhancer for $30 \mathrm{~min}$, then decanting and adding 5\% BSA diluted in PBS spiked with human Fc receptor blocker at $5 \mu \mathrm{L}$ per 1 million cells for $30 \mathrm{~min}$. BSA/FcR blocker was then decanted and primary antibody diluted in 5\% BSA was applied to slides for $1 \mathrm{~h}$. Slides were washed in PBST three times, then incubated in secondary antibodies diluted in PBS for $45 \mathrm{~min}$ in the dark. Whole secondary antibodies were diluted at 1:2500 and $\mathrm{F}(\mathrm{ab})$ fragment secondary antibodies were diluted at 1:800. After washing slides in PSBT three times, DAPI diluted in PBS at $1 \mathrm{mg} / \mathrm{L}$ was added for $5 \mathrm{~min}$ in the dark. Slides were washed in PBST three times before mounting the coverslip using mounting media without DAPI, and were left to cure overnight in the dark.

\section{RareCyte Manual Staining Protocol}

Frozen bone marrow smears on plus slides were thawed on the benchtop at room temperature for 5-10 min, and then fixed in $10 \%$ NBF for $1 \mathrm{~h}$. Slides were washed in TBS 2 times for $5 \mathrm{~min}$, then placed in PBS before performing heat-mediated antigen retrieval in Tris- $\mathrm{HCl}$ buffer, $\mathrm{pH} \mathrm{10}$, for $6 \mathrm{~min}$ at $75^{\circ} \mathrm{C}$. Slides were transferred to fresh PBS for $5 \mathrm{~min}$ to cool, and were then washed four times in Dako buffer (Dako, \#K8007) by directly pipetting buffer onto each slide and decanting. Reagent 1 was then added for $30 \mathrm{~min}$, followed by four washes in Dako buffer. Slides were then incubated in Reagent 2A for $40 \mathrm{~min}$, followed by another set of washes, incubated in Reagent $2 \mathrm{~B}$ for $40 \mathrm{~min}$, and then washed again. Reagent 3 was spiked fresh with anti-CD11b-PE, CD14-PE, and CD34-PE at 1:200 and incubated on slides for $30 \mathrm{~min}$, followed by a final set of washes. Slides were then transferred to PBS and cover-slipped with RareCyte mountant to sit overnight at room temperature in the dark.

\section{Heat-Mediated Antigen Retrieval}

Instead of incubating slides in Triton X-100, slides were placed into microwave-safe containers (PerkinElmer, \#STJAR4) with either citrate-based Antigen Unmasking Solution (Vector Laboratories, \#H-3300), Target Retrieval solution (Dako, \#S1699), or EDTA solution (ThermoFisher, \#005500) diluted to 1X in distilled water. Slides were microwaved at $100 \%$ power for $50 \mathrm{~s}$, and then $20 \%$ power for $15 \mathrm{~min}$. Slides were then placed on the benchtop to cool at room temperature for $15 \mathrm{~min}$.

\section{Manual Imaging of Slides}

Slides were loaded onto a Carl Zeiss AxioImager.Z2 equipped with a PhotoFluor LM-75 halide light source (89 North), a CoolCube $2 \mathrm{~m}$ monochrome camera (MetaSystems, \#H-0310-013-MS), and a motorized 8 slide stage using a manual movement control system (MMC) (MetaSystems, V2.4.5). Images were captured with the Isis Fluorescence Imaging Platform (MetaSystems, V5.8.5) using a Zeiss EC Plan-Neofluar 40×/0.75 M27 objective and a Zeiss Plan-Achromat $20 \times / 0.8$ M27 objective. Slides from the same experiment were imaged using the same settings (exposure time, upper threshold, lower threshold). Nuclear staining with 4'6-Diamidino-2-Phenylindole (DAPI) was visualized using excitation 359 and emission 461 (custom DAPI filter set); AF488 was visualized with excitation 495/ 25 and emission 537/29 (Chroma, \#49303); AF555 was visualized with excitation 550/25 and emission 605/70 (Zeiss, Filter Set $43 \mathrm{HE}$ ); AF647 was visualized with excitation 640/30 and emission 690/50 (Chroma, \#49009).

\section{Imaging Slides Using Metafer Scanning Software}

The number of false and true positive cancer cells in bone marrow slides were counted using the Metafer 5 (MetaSystems, V3.11.8) automated scanning system. Slides were loaded onto the motorized 8 slide stage of the microscope indicated above. Scans were performed using a Zeiss Plan Apochromat $10 \times / 0.45$ objective to scan in the $\mathrm{XY}$ plane, and one $\mathrm{Z}$ focus plane was selected automatically for every tile using a course focus of 10 planes of $7.5 \mu \mathrm{m}$ and then a fine focus of $2 \mu \mathrm{m}$ distance. Slides from the same experiment were scanned using the same area and exposure time for each channel with a 5.0 camera gain. Minimum/maximum exposure times (seconds) for each channel in Fig. 5 were: DAPI 0. 0037/0.0111, AF488 0.0192/0.12, AF555 0.0192/0.04. Exposure times for scans in Fig. 7 were: DAPI 0.0092/0. 0159, AF488: 0.0092/0.24, AF555: 0.0092/0.08.

\section{Metastatic Patient Sample Collection, Processing, and Analysis}

Three patient bone marrow aspirates were collected adjacent to the site of a metastatic prostate cancer lesion (see Additional file 1: Table S3) and were processed onto 
plus slides using the RareCyte system. Slides were stained using the RareCyte manual staining protocol or the optimized protocol. The same defined region was scanned for each slide. Manual selection of the populated gallery after automated scanning detection was performed as in Fig. 5b. The scan could not be completed for Patient 2 BM stained with the RareCyte protocol due to the event limit being reached. Number of total populated cells was thus estimated based on proportion of area that was scanned (see Table 1).

Slides from the same experiment were also subjected to the same criteria for calling candidate $\mathrm{CK}+$ cells. Criteria for manual selection after automated selection is described in the results section. Final cell galleries were exported using Adobe Acrobat.

\section{Results}

\section{Sources of Background Signal}

LNCaP prostate cancer cells spiked into human BM were stained with pan cytokeratin (CK) and CD45 (white blood cell (WBC) marker), resulting in the detection of expected $\mathrm{CK}+\mathrm{WBC}$ - cells but also $\mathrm{CK}+\mathrm{WBC}+$ cells (Fig. 1a). To determine the cause(s) of double positive staining, we cover-slipped and imaged slides after each step in our basic IF protocol [7]. This included imaging after fixation, blocking, primary antibody, secondary antibody only (no primary), and secondary antibody (after primary) steps. We observed autofluorescence immediately after fixation, where a fraction of cells was autofluorescent in every channel, indicating autofluorescence of the BM itself (Fig. 1b). Incubation with unlabeled primary antibody did not further affect signal, but incubation with fluorescently labeled secondary antibody (hereafter referred to simply as secondary antibody) led to substantial signal in a fraction of cells, independent of the BM autofluorescence. In BM samples spiked with cancer cells and stained with both primary and secondary antibodies, CK + WBC- cells were evident as in Fig. 1a. However, the CK staining intensity on these cells was not uniform across all spiked cancer cells, and in some cases, was indistinguishable from the $\mathrm{CK}+$ WBC+ signal. The observed autofluorescence in the initial step after fixation was not surprising, as aldehydebased fixatives are known to produce autofluorescence. Therefore, we decreased the fixation time to $10 \mathrm{~min}$ from $30 \mathrm{~min}$. This was able to reduce autofluorescence without compromising the cells and their subsequent staining (Fig. 1c).

\section{Antigen Retrieval Optimization}

Most automated staining procedures perform heatmediated antigen retrieval in a citrate-based buffer, while in manual staining protocols Triton X-100 is commonly used for cell permeabilization in IF protocols for cells on slides. We compared Triton X-100 to heat-mediated antigen retrieval with three different buffers to determine which produced the best signal-to-noise ratio. We found that permeabilization by Triton X-100 resulted in the best signal-to-noise ratio, as heat-mediated antigen retrieval with any of the buffers produced significantly greater background signal and had decreased true staining intensity (Fig. 2). Furthermore, antigen retrieval with EDTA buffer and target retrieval solution was harsh on the cells, resulting in cell loss and misshapen nuclei.

\section{Blocking Optimization}

The occurrence of background staining after secondary antibody addition indicated an insufficiency in blocking. Our original protocol used 5\% goat serum for blocking, and while this has proven to be sufficient in the detection of CTCs and DTCs in mouse xenograft models, it has been reported that goat serum is ineffective for blocking human samples [22]. Thus, we compared other blocking reagents to improve upon this. In human BM samples stained only with secondary antibody (primary unstained samples), we determined that the best blocking strategies included Image-iT ${ }^{\mathrm{Tr}}$ FX Signal Enhancer, 5\% BSA, and BlockAid ${ }^{\mathrm{m}}$ (Fig. 3a). However, when we used Image-i $T^{\mathrm{im}}$ Signal Enhancer for the blocking step and BlockAid ${ }^{\mathrm{mi}}$ as the antibody diluent (as suggested by the supplier), the background staining increased dramatically (data not shown). We concluded that the combination of an initial blocking step using Image-iT ${ }^{\mathrm{Tx}} \mathrm{FX}$ Signal Enhancer followed by a 5\% BSA block step with $5 \%$ BSA as the primary antibody diluent was the most effective at limiting background signal. Reports have indicated that autofluorescence due to lipofuscin present in macrophages can also contribute to background signal [10-12], so we tested an additional blocking step using TrueBlack $^{\text {tws }}$, which quenches lipofuscin-related autofluorescence. Addition of a TrueBlack ${ }^{\mathrm{Tm}}$ blocking step reduced autofluorescence particularly in the AF555 channel (Fig. 3b).

\section{Secondary Antibody Optimization}

Even though our optimized combination of blocking steps significantly reduced background, there was still a population of BM cells that bound secondary antibodies in the absence of a primary antibody. We hypothesized that this was due to Fc receptor binding, but addition of an Fc blocker did not completely eliminate this nonspecific staining (data not shown). We were able to further reduce this background by diluting the secondary antibody; however, this did not completely eliminate the false signal (Fig. 4) and also decreased true positive signal (Additional file 2: Figure S1). We also reasoned that some non-specific staining could be due to secondary antibody aggregation facilitated by its trimeric structure. 


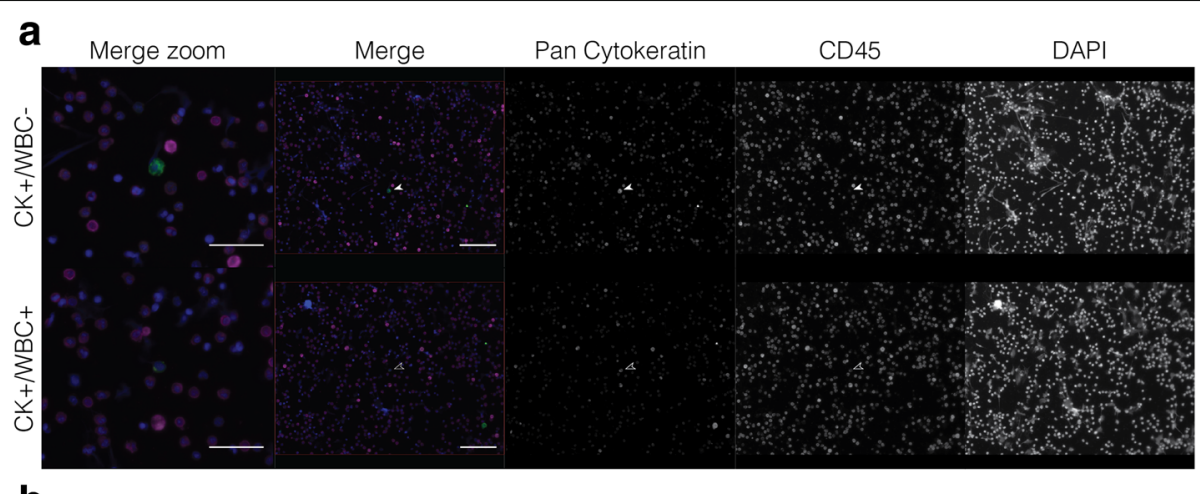

b
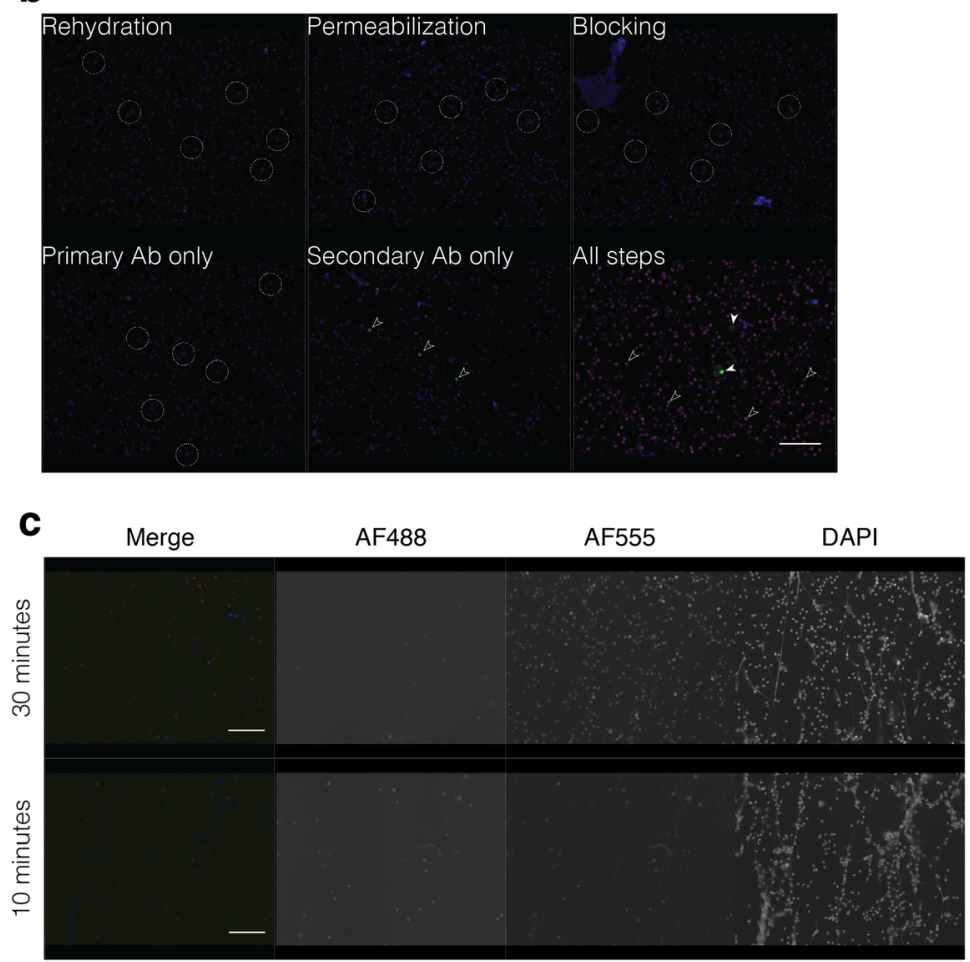

Fig. 1 Identification of sources contributing to background staining in cancer cell-spiked human bone marrow. a Demonstration of true pan cytokeratin+ and white blood cell- (CK + WBC-) putative cancer cell and CK + WBC+ putative WBC using the basic protocol. b Autofluorescence and non-specific secondary antibody binding are evident after rehydration of the slides and secondary antibody incubation, respectively. After all steps are performed, CK staining is detected by AlexaFluor 488 (AF488, green) and CD45 by AF647 (magenta). All images capture signal from all channels, although there is no staining in the AF555 channel. c Autofluorescence in primary unstained bone marrow fixed with 4\% PFA for $30 \mathrm{~min}$ or $10 \mathrm{~min}$. Filled white arrowheads point to true positive staining. Dashed circles indicate background staining on representative cells due to autofluorescence, while open solid arrowheads indicate false staining due to non-specific binding of secondary antibody. Scale bar $=20 \mu \mathrm{m}$ for merge zoom image, $50 \mu \mathrm{m}$ for merged and respective individual channel images in the remainder of the figure

When we used pepsin-digested $F(a b)$ fragment antibodies without the Fc portion, the background signal was further diminished (Fig. 4).

\section{Performance of the Optimized Protocol}

After optimizing every step of the staining protocol, we directly compared it with the basic protocol in cancer cell-spiked and unspiked human BM. We also included primary antibody unstained samples to observe background staining. We found that background signal was significantly reduced using the optimized protocol, and that the overall signal-to-noise ratio was greater (Fig. 5). It was also easier to detect $\mathrm{CK}+\mathrm{WBC}$ - cells by eye when manually scanning the slide. Since manual imaging can be easily manipulated to produce optimal signal, and is not feasible for analyzing large batches of slides to detect rare cells, we then used the Metafer scanning software to scan each slide and populate a gallery of $\mathrm{CK}+$ candidate cancer cells (green) (Fig. 6). For each slide we used the same scan settings, in which we are able to control 


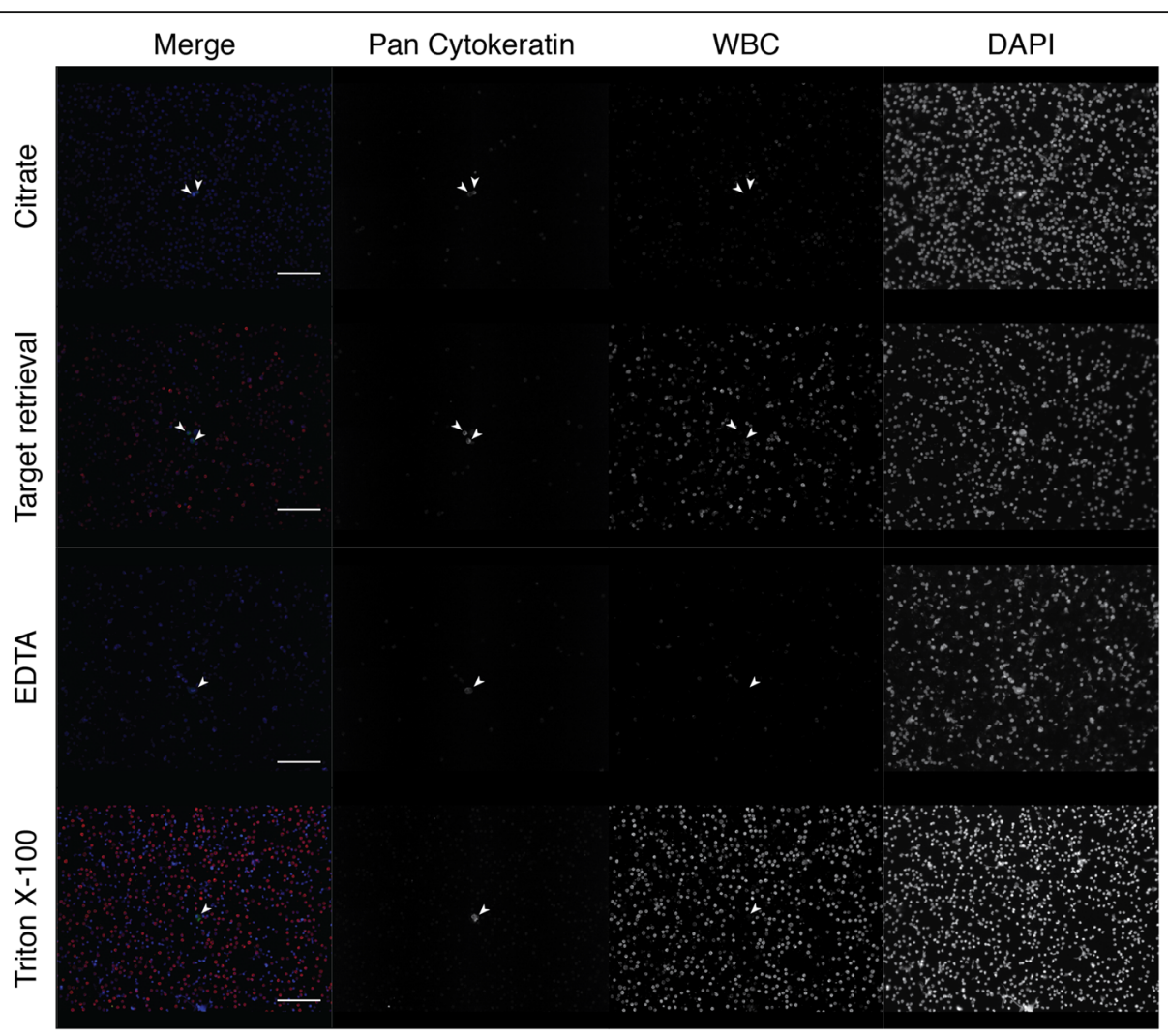

Fig. 2 Comparison of antigen retrieval methods in cancer cell-spiked human bone marrow. Permeabilization using 0.5\% Triton X-100 compared to heat-mediated antigen retrieval using Citrate-based Antigen Retrieval Solution, Target Retrieval Solution, and EDTA solution. White blood cell (WBC) staining represents a cocktail of mouse WBC antibodies (CD45, CD14, CD34, and CD66b) detected by one anti-mouse secondary antibody. Filled white arrowheads point to true positive staining. Scale bar $=50 \mu \mathrm{m}$

the maximum and minimum exposure time for each field, and the signal intensity cutoff for populating the gallery. Exposure time never exceeded $120 \mathrm{~ms}$, and we used lenient cutoff criteria so that low CK-expressing cells would still be detected. Once the gallery was generated we went through three rounds of selection criteria to get rid of extraneous events that the software picked up (Fig. 6). We first eliminated anything that did not visually show up as green. The number of events that was initially detected by the software was almost four times higher when the basic protocol was used, and was still higher after the first screening, indicating that the automated software was picking up background signal.
We then eliminated anything that was clearly not a cell or was not actually green positive after manual observation of the cell. This left us with a gallery of cells that looked to have true positive staining for CK. However, when we assessed the WBC channel (AF555, red) there were cells that were double positive for red and green signal. This was only observed in the slide stained with the basic protocol, and there were more $\mathrm{CK}+\mathrm{WBC}+$ cells (putative WBCs) than CK + WBC- cancer cells (putative cancer cells). On the other hand, in the gallery from the slide that was stained with the optimized protocol, we were easily able to eliminate cells in the first screening step that were not green positive,

Table 1 Cell Counts After Scanning Metastatic Patient BM Stained with the Optimized Protocol or RareCyte Protocol

\begin{tabular}{|c|c|c|c|c|c|c|}
\hline Patient & Protocol & Total populated & First selection & Second selection & $C K+W B C+$ & $C K+W B C$ \\
\hline \multirow[t]{2}{*}{1} & RareCyte & 15,558 & 67 & 29 & 24 & 5 \\
\hline & Optimized & 3775 & 77 & 71 & 51 & 20 \\
\hline \multirow[t]{2}{*}{2} & RareCyte & $>200,000$ & N/A & N/A & N/A & N/A \\
\hline & Optimized & 7318 & 109 & 76 & 20 & 56 \\
\hline \multirow[t]{2}{*}{3} & RareCyte & 9849 & 84 & 40 & 40 & 0 \\
\hline & Optimized & 2774 & 181 & 91 & 49 & 42 \\
\hline
\end{tabular}




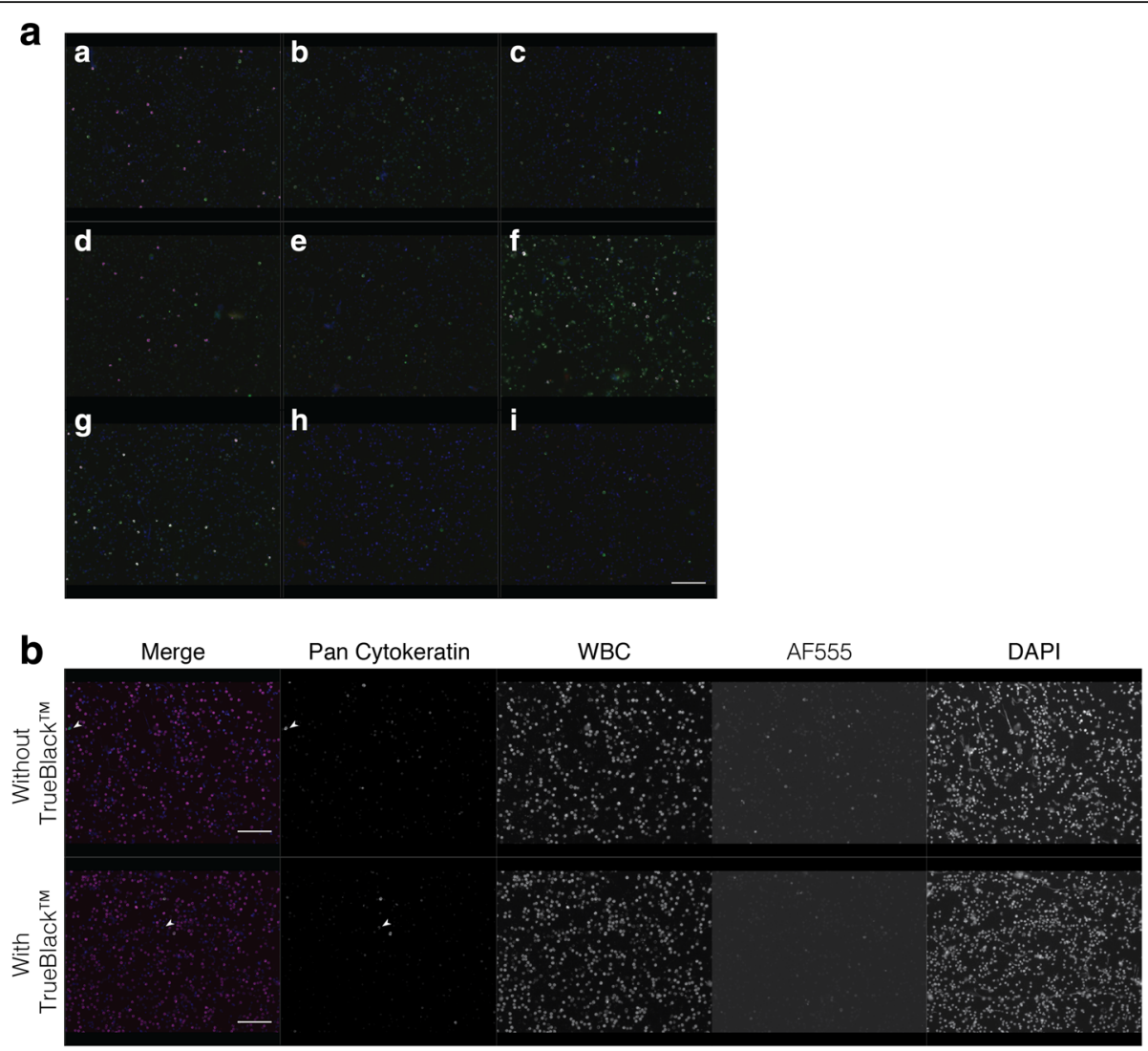

Fig. 3 Optimization of blocking strategy to decrease background signal in cancer cell-spiked human bone marrow. a Comparison of different blocking reagents in primary antibody unstained samples. Goat anti-rabbit AlexaFluor 488 (AF488) and goat anti-mouse AF647 secondary antibodies were applied. Images display merged signal from each fluorescence channel. Blocking reagents used were a: 10\% goat serum; b: 10\% human serum; c: 5\% BSA; d: 10\% goat serum with 5\% BSA; e: 10\% human serum with 5\% BSA; f: Protein Block Serum Free; g: SuperBlock ${ }^{\mathrm{T}}$; h: Image-iT ${ }^{T M}$ FX Signal Enhancer; i: BlockAid ${ }^{T M}$. b Effect of TrueBlack ${ }^{\text {TM }}$ on background signal due to autofluorescence. Samples were stained with pan cytokeratin and white blood cell markers. Autofluorescent signal reduction is most evident in the AF555 channel (where no primary or secondary antibody was applied) and in the AF488 channel (stained for pan cytokeratin). Filled white arrowheads point to true positive staining. Scale bar $=50 \mu \mathrm{m}$

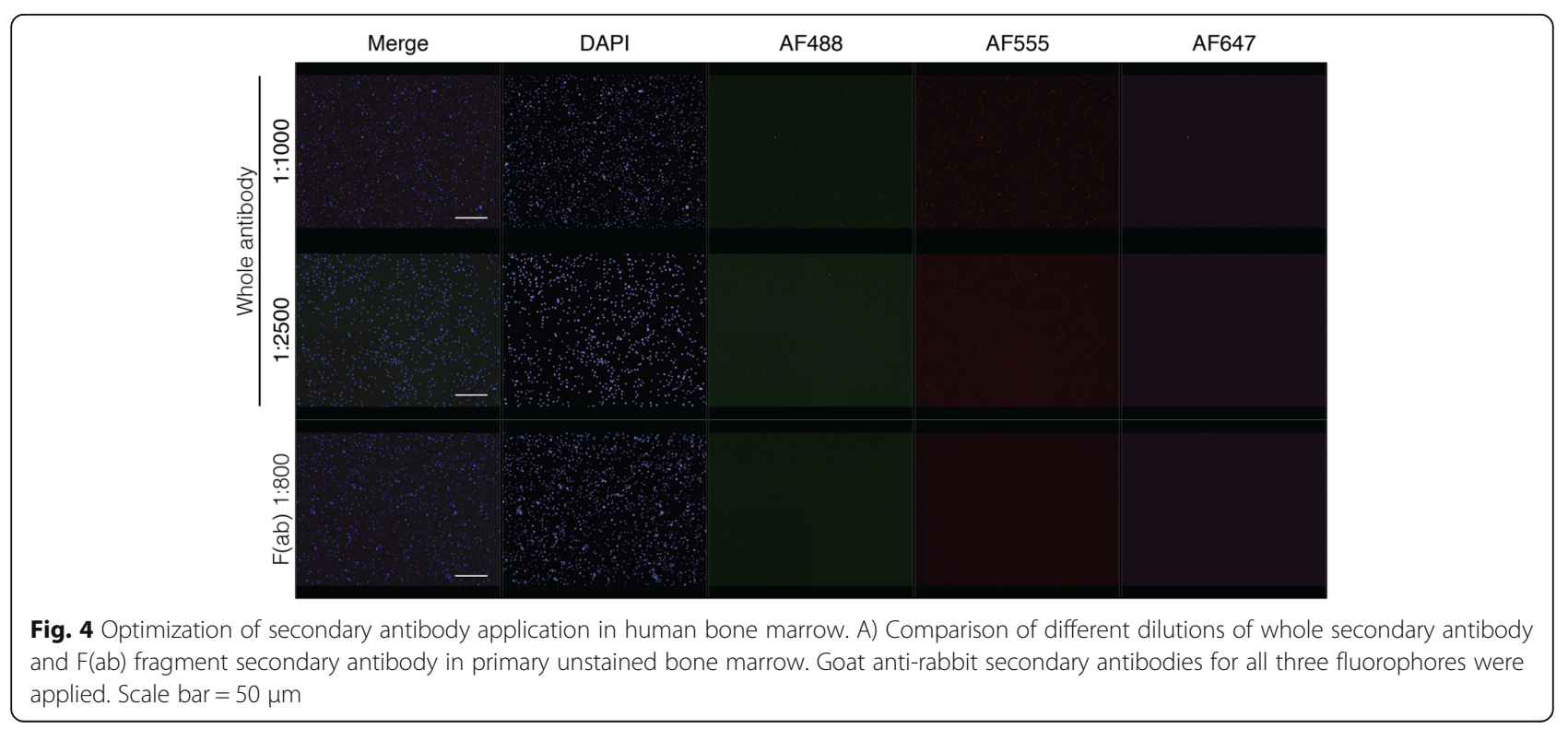




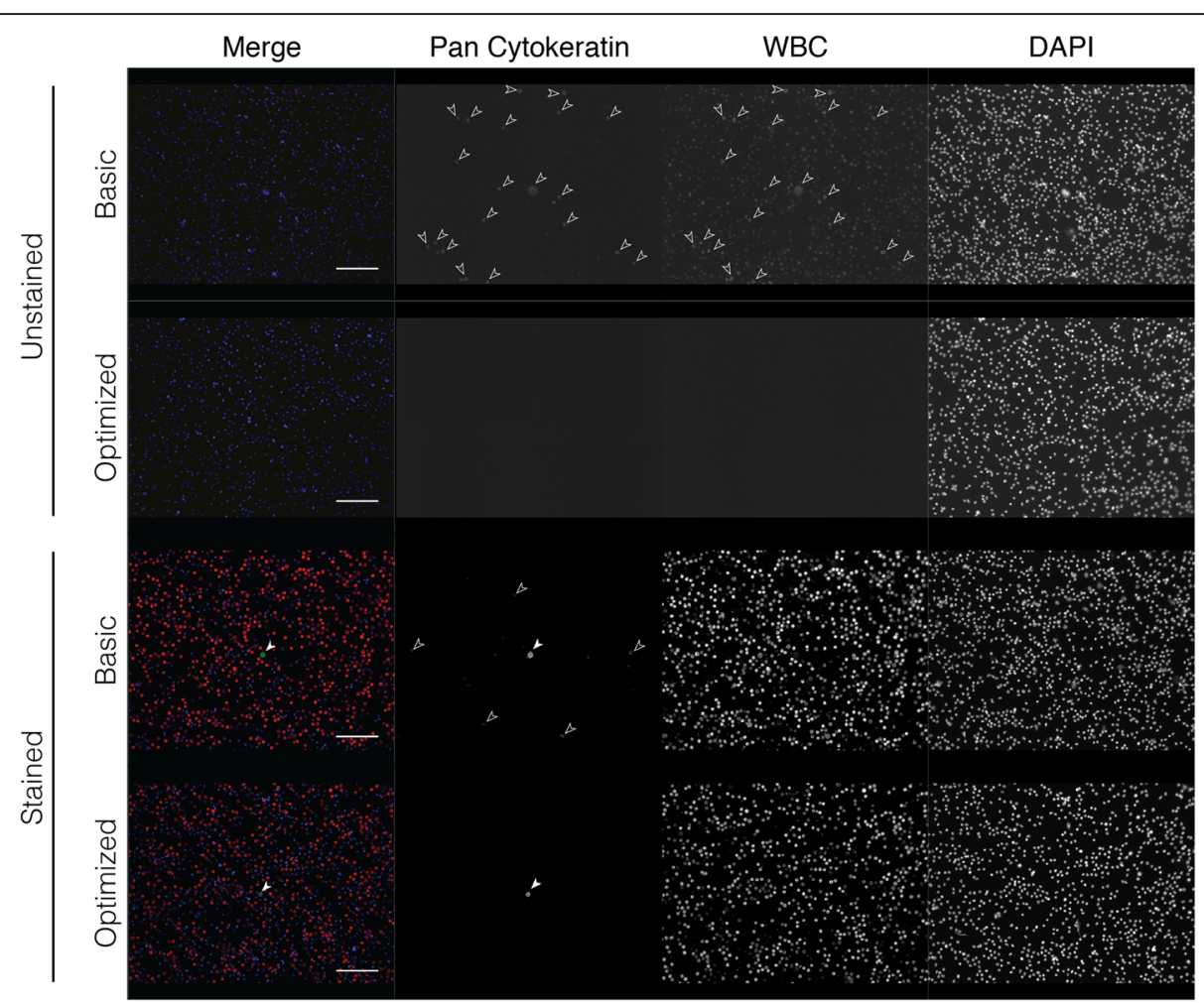

Fig. 5 Comparison of the optimized protocol to the basic protocol. Cancer cell-spiked bone marrow was stained with (stained) and without (unstained) primary antibody using the basic or optimized protocol. Image exposure time was increased for primary unstained slides compared to stained slides, but was consistent between basic and optimized protocols for each. Filled white arrowheads point to true positive staining. Open solid arrowheads indicate false staining due to non-specific binding of secondary antibody. Scale bar $=50 \mu \mathrm{m}$

resulting in only true $\mathrm{CK}+\mathrm{WBC}$ - cancer cells and no double positives or even questionable staining. We then wanted to determine if our optimized protocol had advantages over other established rare cell detection protocols and was compatible with other BM processing techniques. We stained three BM aspirates collected near the site of a metastatic lesion from prostate cancer patients. These samples were collected and processed using the RareCyte AccuCyte ${ }^{\circ}$ system [21]. One of each sample was stained using a manual version of the automated protocol used by RareCyte, which included vastly different reagents and steps compared to ours, or using our optimized protocol. Currently the RareCyte protocol is optimized for use only in blood to detect CTCs, and is not recommended for use in BM. Each slide was scanned using the Metafer detection software to compare the detection of CK + WBC- candidate DTCs between protocols for each patient (Table 1). The higher signal-to-noise ratio observed using the optimized protocol was evident by the decreased number of initial events populated by the gallery and a greater number of candidate DTCs $(\mathrm{CK}+\mathrm{WBC}-)$ for every patient. Overall it was easier to detect candidate DTCs by eye with the optimized protocol, but due to low CK expression in the patient DTCs requiring high exposure times there remained cells that appeared $\mathrm{CK}+\mathrm{WBC}+$ for each protocol (Fig. 7). Our optimized protocol was more sensitive (39.3 average DTCs compared to 2.5 average DTCs) (Table 1) given that each slide for a particular metastatic patient should theoretically have the same number of DTCs.

\section{Discussion}

The ability to predict cancer recurrence before the development of overt metastatic lesions could prevent many deaths due to metastasis. Presently there are no reliable methods to detect recurrent disease before it becomes incurable, but there have been promising developments in liquid biopsy-based rare cell assays. Detection of rare cells by IF staining of BM smears not only allows for quantification, but allows for their biological characterization, which will be crucial in guiding treatment strategies. Unfortunately, there is no widely accepted staining protocol that has the necessary specificity and sensitivity criteria to accurately detect $\sim 1$ cancer cell out of ten million BM cells. It is imperative that procedures intended for clinical use like this be standardized and rigorously optimized, as they are meant to inform important diagnostic and prognostic decisions. The closest "gold standard" is an FDA-approved IF-based detection platform called 


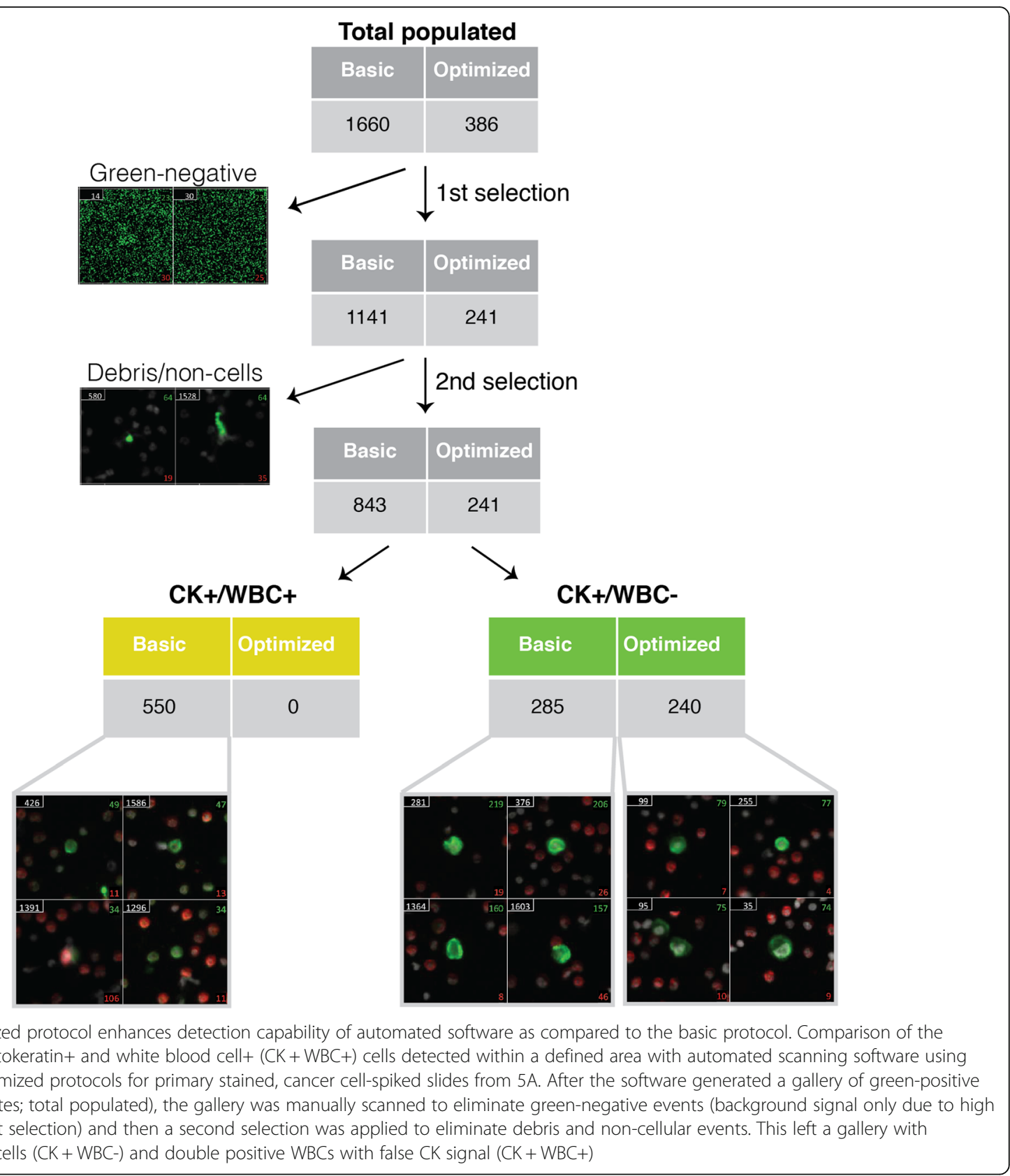

CELLSEARCH [6]. However, it is only approved for CTC detection in blood and relies solely on the epithelial markers EpCAM and cytokeratin. While the detection of CTCs using other markers and platforms have also been somewhat successful, no method for BM DTC detection has been successful [5]. Since it is more informative to understand the biology of a "successful DTC" in the BM compared to the many hundreds of CTCs that enter the circulation but do not survive, it is essential that a standard procedure for IF staining of the BM be developed [23]. The lack of platforms for DTC detection is due to the increased immune component, increased autofluorescence, and difficulty of staining samples in the BM compared to in the blood. The consequences of this are two-fold: 1) more immune cells produce more false positive staining, and 2) markers used to detect CTCs may no longer be specific for DTCs because they might also be expressed by a particular population of BM cells. In order to accurately assess specific detection markers for rare cells, it is critical that any false staining attributed to the autofluorescent nature and large immune component of the BM be eliminated.

In our experience with staining BM for CK to detect cancer cells, we have found that primary antibody unstained cancer-free samples contain cells that have 


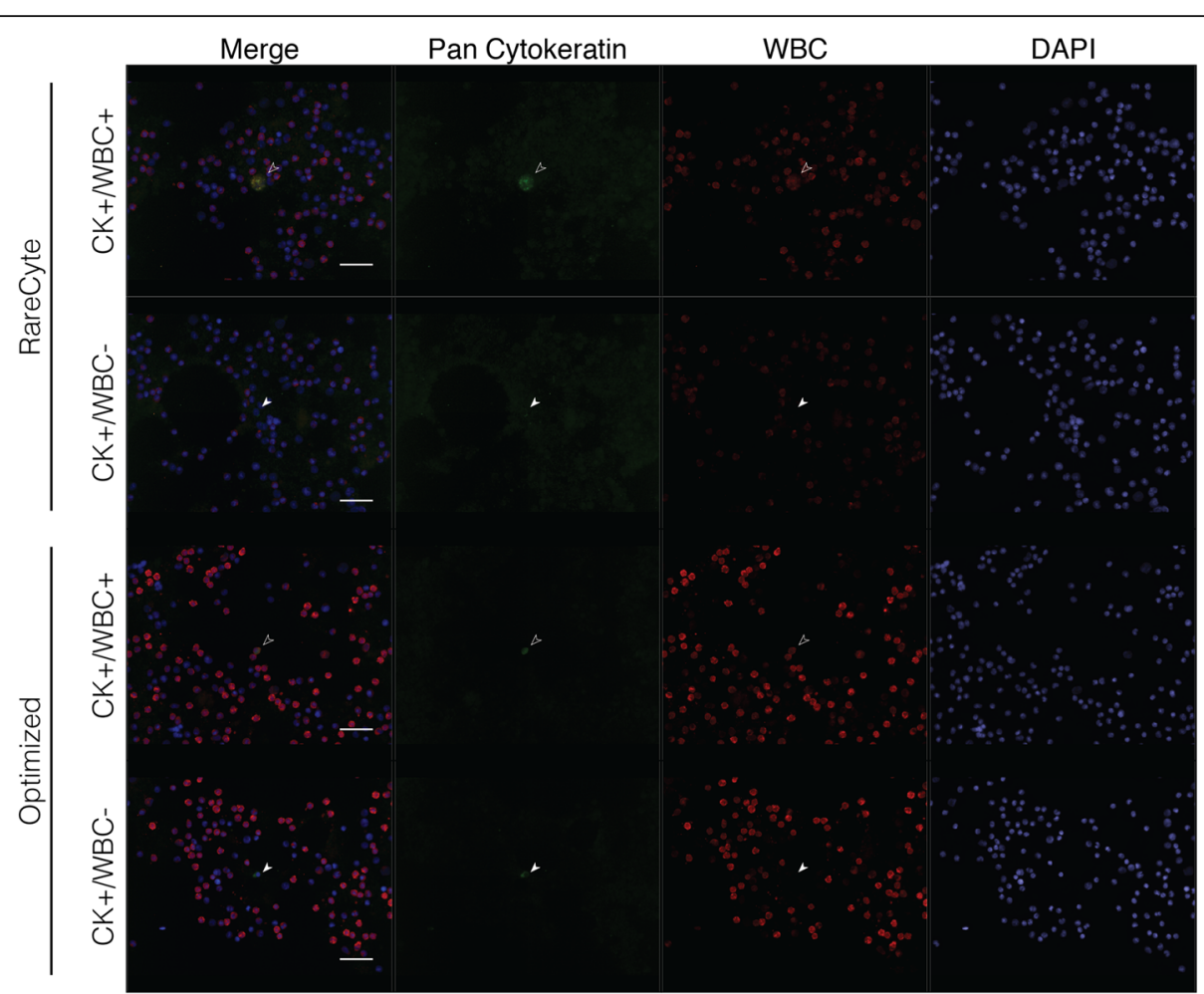

Fig. 7 The optimized protocol enhances detection of patient DTCs compared to alternately stained and processed slides. Selected images representing candidate disseminated tumor cells $(C K+W B C-)$ and $C K+W B C+$ cells from Patient 1 in Table 1 stained with the RareCyte or optimized protocol. Image exposure time and brightness and contrast settings had to be altered between the slides stained with the optimized and the RareCyte protocol due to a decrease in overall signal with the RareCyte protocol. Scale bar $=40 \mu \mathrm{m}$

positive signal and are indistinguishable from real signal in cancer cell line spiked samples by automated detection software. It is possible that some BM cells can express CK; however, the positive control cancer cells used here are distinguishable by their high frequency and staining pattern. The cells found in cancer-negative controls display spatially overlapping signal in each channel with no distinct pattern, whereas true cytoplasmic filamentous CK staining should only be detectable in one pre-determined channel. In this study, we were able to identify and eliminate the sources contributing to the ambiguous background signal: autofluorescence and nonspecific binding of fluorescently-labeled secondary antibody. Other studies have observed these background signals in BM as well, citing macrophages and neutrophils as major culprits $[9,24]$. Both sources of background were visible in every channel, but autofluorescence was the strongest in the AF555 channel. We were able to decrease this BM tissue autofluorescence by reducing fixation time from 30 to $10 \mathrm{~min}$, including a TrueBlack ${ }^{\mathrm{Tm}}$ blocking step, and using Triton X-100 permeabilization over heatmediated antigen retrieval methods. To reduce nonspecific binding of secondary antibodies (the main source of strong background signal), we combined blocking with 5\% BSA and Fc receptor block with the Image-iT ${ }^{\mathrm{tm}}$ Signal
Enhancer. To amplify true signal we used indirect labeling with primary and secondary antibodies rather than fluorophore-conjugated primary antibodies. We found that the use of $F(a b)$ fragment secondary antibodies was helpful in reducing background signal for $\mathrm{CK}$, but that the use of whole secondary antibodies boosted the signal of WBCs (Additional file 2: Figure S1). These steps collectively increased true signal and reduced background signal, allowing for shorter exposure time and a higher signal-tonoise ratio. Other conditions we found to enhance signalto-noise ratio but that we did not test directly included washing and antibody incubation temperature and time. We extended wash times and used PBST instead of PBS, and performed all antibody incubations at room temperature instead of at $37^{\circ} \mathrm{C}$, which was previously recommended [18]. By changing these parameters, we were able to eliminate all significant background contributing to high false CK signal on immune cells.

Due to the rarity of DTCs, the use of automated scanning microscopy is required for their detection and enumeration. We compared our novel optimized IF protocol to our previous basic IF protocol, as well as to a CTC detection protocol from RareCyte. When we stained cancer cell-spiked BM with our optimized protocol, we were able to detect the same number of $\mathrm{CK}+\mathrm{WBC}$ - putative cancer 
cells as the basic protocol, but with a significant reduction in manual screening time (ten minutes compared to three hours). This was due to an enormous reduction in extraneous events, caused by high background. When we stained advanced cancer patient BM with our optimized protocol compared to the RareCyte protocol (optimized for blood, not BM), we detected more CK + WBC- putative DTCs. We also observed an impressive reduction in background signal, which allowed for significantly less manual screening time. In these patient samples we observed more extraneous events and $\mathrm{CK}+\mathrm{WBC}+$ cells than in the cell-line spiked BM (Table 1); this is likely due to lower CK expression in the actual patient DTCs relative to the cancer cell line, so a much higher exposure time was required. In some cancers, however, CK expression is considerably higher, so the signal-to-noise ratio is much greater (Additional file 3: Figure S2). CK heterogeneity was also observed between cancer cells in the cancer cell line and in patient samples. The purpose of our study was to eliminate background signal contributing to inconsistencies and uncertainty when testing cancer-specific markers. To this end, our optimized protocol 1) increased signal-to-noise, 2) decreased extraneous events picked up by automated detection software, 3) can be applied to variously prepared samples, and 4) detected patient DTCs. Ongoing work is being done to adapt this optimized protocol for use with automatic staining devices for high volume processing, a requirement for rare cell detection.

\section{Conclusions}

In this study, we have optimized the staining conditions for BM smears in order to control and minimize signal due to factors other than the expression of the candidate cancer cell marker. Our optimization increased the signal-to-noise ratio and eliminated sources of false staining. This assay can now be used to investigate the sensitivity and specificity of individual candidate diseasespecific markers (e.g. prostate specific antigen for prostate cancer), as any observed signal can be confidently qualified as a true positive signal. Finally, we are working to automate this assay for use with high volume clinical samples in order to detect and characterize CTCs and DTCs to predict cancer recurrence.

\section{Additional files}

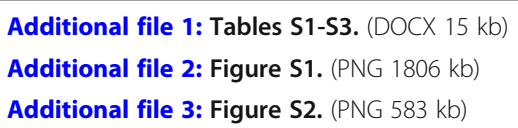

\section{Abbreviations}

BM: Bone marrow; CTC: Circulating tumor cell; DTC: Disseminated tumor cell; Fc: Fragment crystallizable; IF: Immunofluorescence; WBC: White blood cell

\section{Acknowledgements}

The authors would like to thank Emily Caruso and Stephanie Glavaris for obtaining voluntary consent from patients and collecting bone marrow samples. Thanks to Jessica Hicks for her expert advice in immunostaining. Finally, thanks to all of the members of the Pienta Lab for their feedback during the optimization process

\section{Funding}

This study was supported by the Prostate Cancer Foundation, the Patrick C. Walsh Prostate Cancer Research Fund, NIH Grant F32-CA206394, and NIH Grant P01-CA093900.

\section{Availability of Data and Materials \\ Not applicable.}

\section{Authors' Contributions}

$\mathrm{HA}$ and $\mathrm{KV}$ designed experiments, interpreted results, and processed bone marrow samples. HA performed the IF staining, imaging, and automated scanning and counting with help from KV. HA wrote the manuscript and KV and KP reviewed and edited the manuscript. All authors read and approved the final manuscript.

\section{Ethics Approval and Consent to Participate}

Bone marrow was collected from patients following signed written formal consent approved by the Johns Hopkins Office of Human Subjects Research Institutional Review Board.

\section{Consent for Publication}

Not applicable

\section{Competing Interests}

The authors declare that they have no competing interests.

\section{Publisher's Note}

Springer Nature remains neutral with regard to jurisdictional claims in published maps and institutional affiliations.

Received: 15 November 2017 Accepted: 3 April 2018

Published online: 01 July 2018

References

1. Mundy GR. Metastasis to bone: causes, consequences and therapeutic opportunities. Nat Rev Cancer. 2002;2:584-93.

2. Chambers AF, Groom AC, MacDonald IC. Dissemination and growth of cancer cells in metastatic sites. Nat Rev Cancer. 2002;2:563-72.

3. Massague J, Obenauf AC. Metastatic colonization by circulating tumour cells. Nature. 2016;529:298-306.

4. van der Toom EE, Verdone JE, Gorin MA, Pienta KJ. Technical challenges in the isolation and analysis of circulating tumor cells. Oncotarget. 2016;7: 62754-66.

5. van der Toom EE, Verdone JE, Pienta KJ. Disseminated tumor cells and dormancy in prostate cancer metastasis. Curr Opin Biotechnol. 2016:40:9-15.

6. Allard WJ, Matera J, Miller MC, Repollet M, Connelly MC, Rao C, et al. Tumor cells circulate in the peripheral blood of all major carcinomas but not in healthy subjects or patients with nonmalignant diseases. Clin Cancer Res. 2004;10:6897-904.

7. Valkenburg KC, Amend SR, Verdone JE, van der Toom EE, Hernandez JR, Gorin MA, et al. A simple selection-free method for detecting disseminated tumor cells (DTCs) in murine bone marrow. Oncotarget. 2016:7:69794-803.

8. Hulspas R, O'Gorman MR, Wood BL, Gratama JW, Sutherland DR. Considerations for the control of background fluorescence in clinical flow cytometry. Cytometry B Clin Cytom. 2009;76:355-64.

9. Li F, Yang M, Wang L, Williamson I, Tian F, Qin M, et al. Autofluorescence contributes to false-positive intracellular Foxp3 staining in macrophages: a lesson learned from flow cytometry. J Immunol Methods. 2012;386:101-7.

10. Clark KG, Davidson WM. Bone marrow lipofuscin. J Clin Pathol. 1972;25:947-50.

11. Hohn A, Grune T. Lipofuscin: formation, effects and role of macroautophagy. Redox Biol. 2013;1:140-4.

12. Jain SK, Abreo K, Duett J, Sella ML. Lipofuscin products, lipid peroxides and aluminum accumulation in red blood cells of hemodialyzed patients. Am J Nephrol. 1995;15:306-11. 
13. Prentice Al. Autofluorescence of bone tissues. J Clin Pathol. 1967;20:717-9.

14. Zoumi A, Yeh A, Tromberg BJ. Imaging cells and extracellular matrix in vivo by using second-harmonic generation and two-photon excited fluorescence. Proc Natl Acad Sci U S A. 2002:99:11014-9.

15. Zipfel WR, Williams RM, Christie R, Nikitin AY, Hyman BT, Webb WW. Live tissue intrinsic emission microscopy using multiphoton-excited native fluorescence and second harmonic generation. Proc Natl Acad Sci U S A. 2003:100:7075-80.

16. Ranjit S, Dvornikov A, Stakic M, Hong SH, Levi M, Evans RM, et al. Imaging fibrosis and separating collagens using second harmonic generation and phasor approach to fluorescence lifetime imaging. Sci Rep. 2015;5:13378.

17. Miyamoto DT, Sequist LV, Lee RJ. Circulating tumour cells-monitoring treatment response in prostate cancer. Nat Rev Clin Oncol. 2014;11:401-12.

18. Marrinucci D, Bethel K, Kolatkar A, Luttgen MS, Malchiodi M, Baehring F, et al. Fluid biopsy in patients with metastatic prostate, pancreatic and breast cancers. Phys Biol. 2012;016003:9.

19. Moreno JG, O'Hara SM, Gross S, Doyle G, Fritsche H, Gomella LG, et al. Changes in circulating carcinoma cells in patients with metastatic prostate cancer correlate with disease status. Urology. 2001;58:386-92.

20. Deng G, Herrler M, Burgess D, Manna E, Krag D, Burke JF. Enrichment with anti-cytokeratin alone or combined with anti-EpCAM antibodies significantly increases the sensitivity for circulating tumor cell detection in metastatic breast cancer patients. Breast Cancer Res. 2008;10:R69.

21. Campton DE, Ramirez AB, Nordberg JJ, Drovetto N, Clein AC, Varshavskaya $P$, et al. High-recovery visual identification and single-cell retrieval of circulating tumor cells for genomic analysis using a dual-technology platform integrated with automated immunofluorescence staining. BMC Cancer. 2015;15:360

22. Buchwalow I, Samoilova V, Boecker W, Tiemann M. Non-specific binding of antibodies in immunohistochemistry: fallacies and facts. Sci Rep. 2011;1:28

23. de Groot AE, Roy S, Brown JS, Pienta KJ, Amend SR. Revisiting seed and soil: examining the primary tumor and Cancer cell foraging in metastasis. JEMol Cancer Res. 2017:15:361-70.

24. Watt SM, Burgess AW, Metcalf D, Battye FL. Isolation of mouse bone marrow neutrophils by light scatter and autofluorescence. J Histochem Cytochem. 1980;28:934-46.

\section{Ready to submit your research? Choose BMC and benefit from:}

- fast, convenient online submission

- thorough peer review by experienced researchers in your field

- rapid publication on acceptance

- support for research data, including large and complex data types

- gold Open Access which fosters wider collaboration and increased citations - maximum visibility for your research: over $100 \mathrm{M}$ website views per year

At BMC, research is always in progress.

Learn more biomedcentral.com/submissions 